\title{
Metal Fatigue Features
}

\section{P. Brož}

This paper presents a summary of fatigue, crack initiation and growth, and fractographic findings for metal materials. The purpose of this paper is to consolidate and summarize some aspects of the fatigue of materials and structures.

Keywords: fracture surface, fracture toughness, macro-and microscopic appearance, microcrack, slip band, small cracks, striation, subgrain, transcrystalline.

\section{Introduction}

A major problem confronting the designer is to choose the correct materials for the design of an individual component or structure; the material properties have to ensure that the component performs the work for which it was designed without malfunctioning throughout its guarranted life, and can be produced for a price acceptable to the customer.

To enable this, the designer needs to know the loads that his component or structure will be subjected to the environment in which it will act, the service life expected, and the production cost. These factors limit the range of materials for application in the distinctive design. To finalize the selection of materials, he needs to be aware of their characteristics under various loadings and in various environments (i.e. the material properties). Knowing these properties, he must be able to correlate them with the bearing capacity of his proposed component or structure. Fracture mechanics deals with these questions. Conventional design assumes that the material is a flawless continuum. However, we know that rational design and material evaluation require knowledge of flaws in materials. Many materials, components and structures either have inherent defects as part of the production routine, or develop them at some phase of their life. Comprehension of how a cracked body conducts itself under loading is fundamental to the explanation of any fatigue problem. Since much of the information on fatigue from both laboratory experiments and service characteristics was obtained and expounded before the study of cracked-material behaviour was implemented, no established field of study correlated the particulars. Isolated sets of data have practically become fatigue folklore, and their relation to other groups of data has not been clarified.

\section{Crack initiation}

Significant publications on fatigue research, in the $20^{\text {th }}$ century include Forsyth [1], on extrusions and intrusions in slip bands, see Fig. 1. Three basic observations are: the importance of the free material surface, the irreversibility of cyclic slip, and environmental influences on microcrack initiation. For the most part, microcracks begin at the free material surface, and in unnotched specimens possessing a nominally homogeneous stress distribution loaded with cyclic tension. There is less prevention of cyclic slip than inside the material for the free surface at one side of the surface material. Microcracks also start more easily in slip bands with slip displacements normal to the material surface [2]. There remain questions about why cyclic slip is not reversible. As far back as the $1950 \mathrm{~s}$, it was understood that there are two reasons for non-reversibility. One argument is that (cyclic) strain hardening occurs, which implies that not all dislocations return to their original position. Another important factor is the interaction with the environment. A slip step at the free surface implies that fresh material is exposed to the environment. In a non-inert environment, most technical materials are rapidly covered with a thin oxide layer, or some chemisorption of foreign atoms of the environment occurs. Exact reversibility of slip is then obviated. Fatigue initiation is a surface effect.

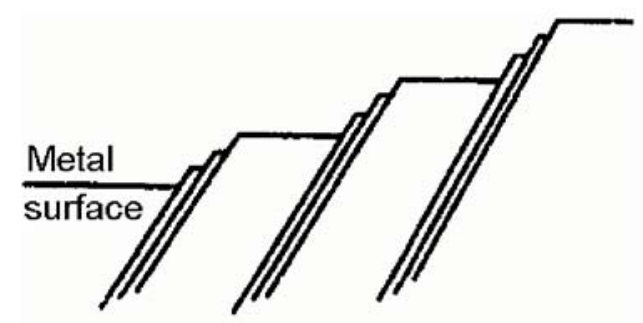

Steady stress

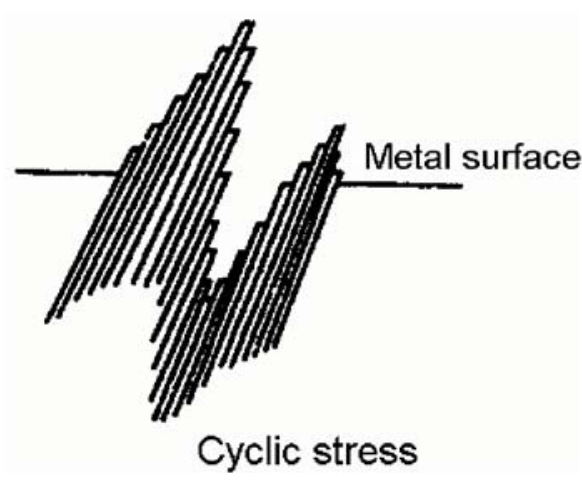

Fig. 1: Slip geometry at the material surface according to Forsyth [1]

In the mid $20^{\text {th }}$ century, microscopic investigations were still made with an optical microscope. This implies that crack nucleation was observed on the surface, where it indeed occurs. As soon as cracks grow into the material away from the free surface, only the ends of the crack front can be observed on that free surface. It is questionable whether this information is representative of the growth process inside the material, a problem that is sometimes overlooked. Microscopic observations on crack growth inside the material require cross-sections of the specimen are made. Several investiga- 
tions employing sectioning were made in the 1950s and earlier. These showed that in most materials fatigue cracks grow transcrystalline. Although the fatigue fractures appeared rather flat to the unaided eye, it turned out that the crack growth path under the microscope could be rather irregular, depending on the type of material. In materials with low stacking fault energy (e.g. Cu- and Ni-alloys), cross slip is difficult and as a result the cyclic slip bands are narrow and straight. Crack growth on a microscale occurs in straight segments along these bands. In materials with high stacking fault energy (e.g. Al-alloys) cross slip is easy. Moreover, in the $\mathrm{Al}$ crystal lattice there are many slip systems which can easily be activated. As a consequence, the slip lines are wider and can be rather wavy. Crack growth on a micro scale does not suggest that it occurs along crystallographic planes. As a result, fatigue on a microscale can be significantly different for different materials. The behavior is structure-sensitive, depending on the crystal structure (fcc, bcc, or hexagonal), the elastic anisotropy of the crystalline structure, grain size, texture, and dislocation obstacles (e.g. pearlite bands in steel, precipitated zones in $\mathrm{Al}$-alloys, twins, and so on).

The outset of the damage in a cyclically stressed metal is tied in with a free surface. There is the following evidence that damage in a polycrystalline ductile metal is connected with grains having a free surface rather than those within the body:

(i) Surface grains are in intimate touch with the atmosphere; thus if the environment is a factor in the damage process, they are apparently more receptive. A surface grain is the only part of a polycrystal not fully supported by adjoining grains. Because the slip systems in neighbouring grains of a polycrystal are not related to each other, a grain having a free surface will be able to deform plastically more easily than a grain in the body of the metal that is surrounded by other grains.

(ii) It has been shown that if a fatigue test is stopped after some fraction (perhaps 20 per cent) of the expected life of the specimen, a thin layer of metal is removed from the test section, and the test is continued at the same stress level, the total life of the specimen is longer than the expected life of the original specimen. If a surface layer is removed at frequent intervals throughout a test, the expected life may be exceeded many times; in fact, provided that the stress amplitude is maintained constant and the frequency of removal and the depth of the removed layer are sufficient, the life will be limited only by the initial cross-sectional area of the specimen.

(iii) The fatigue strength of small specimens cut from the interior of the test-section of a larger specimen broken in reversed direct stress (that is, cut from material which has been subjected to a stress level greater than the plain fatigue limit) is not lower than that of the virgin material.

(iv) If the surface of a specimen is hardened, either metallurgically or by surface working, the fatigue strength of the specimen en bloc may be increased. Similarly, any procedure that softens the surface decreases the fatigue strength of the specimen.

(v) Metallurgical examination of broken fatigue specimens of nominally homogeneous metallic specimens which have been subjected to a uniform stress distribution over their cross-section does not reveal cracks in the body of the specimen. In certain circumstances, however, cracks may form in the interior of a specimen at inclusions or flaws or below hardened surface layers.

The onset of damage and cracking is thus associated with the surface grains, only those grains in the body of a specimen through which a crack, formed in a surface grain, passes as it grows across the specimen being damaged. This means that it is relatively simple to make a direct observation of the progressive development of cracking during a fatigue test. In general, only one crack penetrates into the metal to any considerable depth, but many additional cracks may be visible to the naked eye on the surface of soft metals (for example, copper, mild steel), especially when tested at stress levels giving failure after relatively short endurance (say, less than $10^{5}$ cycles). On the other hand, no cracks, with the exception of the crack leading to complete failure, may be visible to the naked eye on the surface of specimens of hard metallic alloys such as high strength steels and aluminium alloys, which are light and hardenable.

\section{Fractographic features}

A milestone in experimental research was the introduction of the electron microscope (EM), originally the transmission electron microscope (TEM) in the 1950s, and later the scanning electron microscope (SEM) in the 1970s. Microscopic investigations in the TEM are more laborious than in the SEM, because it is necessary to make either a replica of the fracture surface, or a thin foil of the material. The thin foil technique is destructive and does not show the fatigue fracture surface. However, information on the material structure can be obtained, such as the formation of subgrains under cyclic loading.

Investigations of fatigue fracture surfaces in the SEM are now a rather well standardized experimental alternative which can indicate where the fatigue fracture started, and in which directions it was growing [2]. A fundamental observation was made with the electron microscope around 1960. Fractographic pictures revealed striations which could be correlated with individual load cycles. By mixing small and large load cycles in a fatigue test the occurrence of one striation per load cycle was proven by Ryder [5]. The striations are assumed to be remainders of microplastic deformations at the crack tip, but the mechanism can be different for different materials. Because of microplasticity at the crack tip and the crack extension mechanism in a cycle, it should be expected that the profile of the striations depends on the type of material. Terms such as ductile and brittle striations were adopted. Striations could not be observed in all materials, at least not equally clearly. Moreover, the visibility of striations also depends on the severity of the load cycles. At very low stress amplitudes it may be difficult to see striations, although fractographic indications were obtained which showed that crack growth still occurred in a kind of cycle-by-cycle sequence.

Striations have also shown that the crack front is not simply a single straight line, as usually assumed in fracture mechanics analysis. Noteworthy observations on this problem were made by Bowles in the late 1970s [6]. Bowles developed a vacuum infiltration method to obtain a plastic casting of the 


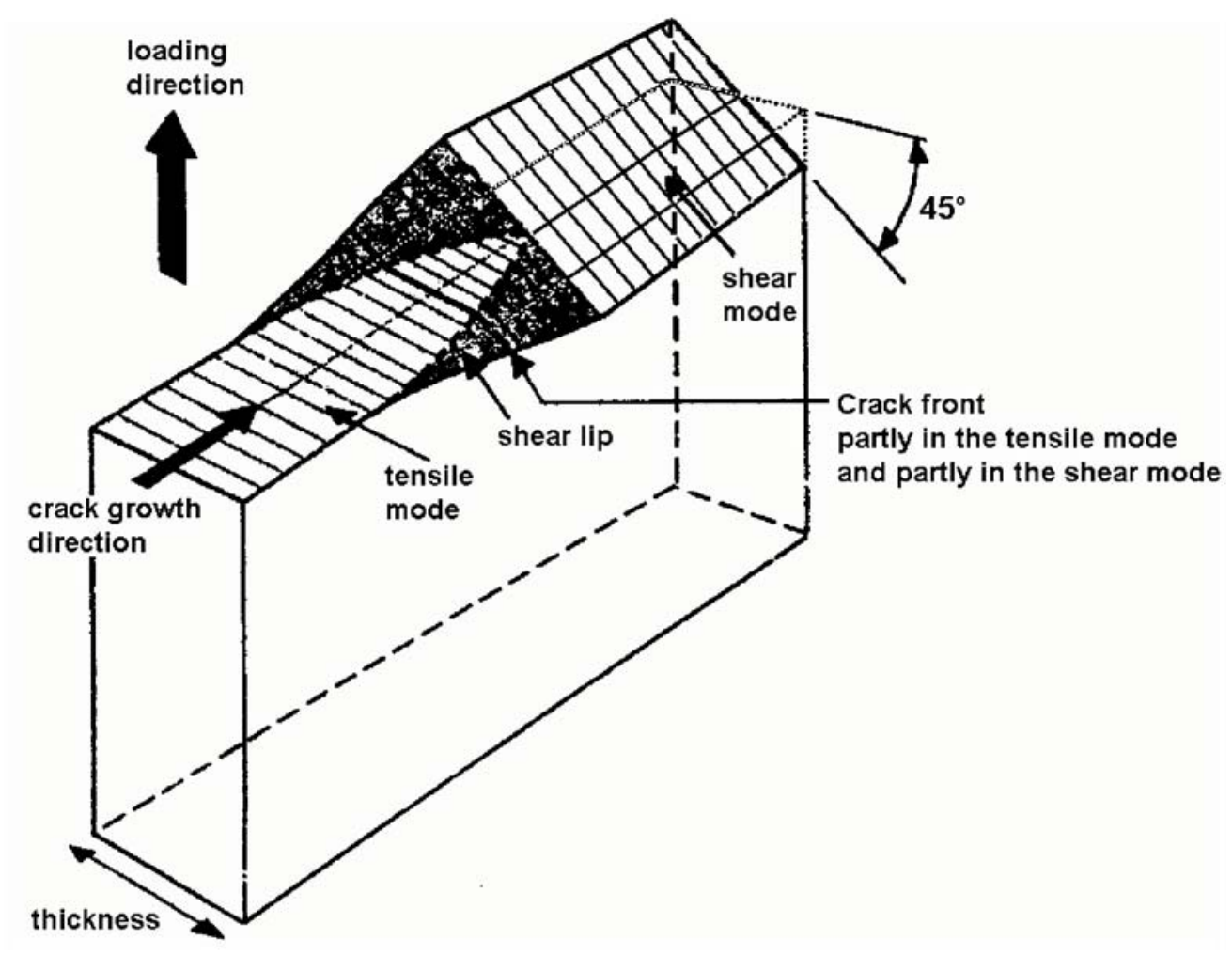

Fig. 2: Fatigue crack growth with a transition from tensile mode to shear mode (taken from [2])

entire crack. The casting could then be studied in the electron microscope.

Macroscopic shear lips, see Fig. 2, were well known for aluminium alloys from the early 1960s, but they were also observed on fatigue cracks in other materials. The width of the shear lips increased for faster fatigue crack growth, and finally a full transition from a tensile mode fatigue crack to a shear mode fatigue crack can occur. The shear lips are a surface phenomenon because crack growth in the shear mode is not so constrained in the thickness direction. Shear lips are macroscopic deviations from a mode I crack assumed in a fracture mechanics analysis.

Fatigue cracks in thick sections can be found largely in the tensile mode (mode I) because shear lips are then relatively small. However, the topography of the tensile mode area observed in the electron microscope indicates a more or less uneven surface, although it appears rather flat if viewed with the unaided eye. Large magnifications clearly show that the fracture surface on a microlevel is not at all a nicely flat area. It is a rather irregular surface going up and down in some random way depending on the microstructure of the material. An inert environment increases the surface roughness, whereas an aggressive environment (salt water) promotes a smoother fracture surface. Similarly, the shear lips are narrower in an aggressive environment and wider in an inert environment. These trends are associated with the idea that an aggressive environment stimulates tensile-decohesion at the crack tip, whereas an inert environment promotes shear decohesion. It should be understood that the crack extension in a cycle (i.e. the crack growth rate) depends on the crack growth resistance of the material, and also on the crack driving force, which is different if deviations of the pure mode I crack geometry are present, e.g. shear lips and fracture camber.

Since the mid $20^{\text {th }}$ century, much work has been done on investigating the microscopic incidence of fatigue fracture surfaces, using the electron microscope. Various collections of electron micrographs demonstrating the particulars of the fracture faces created by a growing fatigue crack have been published. The most dominant features of fatigue fracture surfaces (especially those performed by cracks growing on $90^{\circ}$ planes) are distinct line markings, parallel to each other and normal to the crack growth direction. These are known as striations; each striation is in accordance with one load cycle. As a rule, striations are more clearly defined in ductile than brittle materials; e.g., in more resistant steels, the striations are short and discontinuous and their successive positions are not explicitly defined.

The presence of striations on a fracture surface is proof that the failure was caused by fatigue [7], but they cannot always be found on all fatigue fracture surfaces, often because the microscope used has insufficient resolution. Striations varying in spacing from about $2.5 \mathrm{~mm}$ [8] to less than $2.5 \times 10^{-5} \mathrm{~mm}$ [9] have been observed on various materials. At high crack growth rates they tend to give way to ductile dimples [10].

On a microscopic scale, fatigue crack growth is often an irregular process. A study [11] of the shape of the front of a crack growing in a $3.2 \mathrm{~mm}$ thick mild steel sheet was made by examining sections on planes normal to the direction of crack growth so as to eventually intersect the crack front. This showed that the crack front bows forward slightly so that a section can be made with the leading part of the crack in the 
middle of the sheet thickness. In the region of the crack front, numerous apparently independent cracks were found (see Fig. 3).

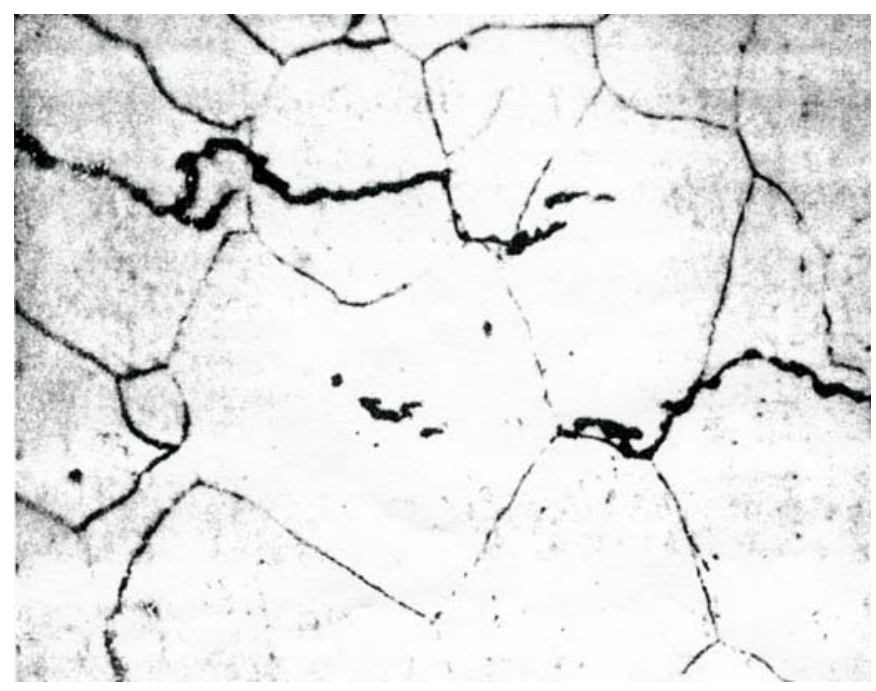

Fig. 3: Section ahead of main crack front in mild steel sheet (taken from [10])

The one-to-one correspondence between striations and applied loads was first proved by Forsyth and Ryder [7]. Microhardness tests taken on the mild steel specimens indicated that a small region at the apex of the rounded-off fatigue crack was as hard as the cup part of a static tensile fracture, just as in the case of the tips of cracks that had been sharpened by compression.

The deformation produced at the crack tip during each cycle resulted in an extension of the crack, crack propagation being a continuous process and resulting from a miniature double-cup plastic separation repeated each cycle.

Fig. 4 (a) shows an example of a blunted tip having two ears, found in a mild steel specimen in which the crack was filled with plastic steel while the maximum load was held constant. However, other sections revealed that the crack tip could be either blunted with only one ear or no ears, or it could still retain a sharp profile as shown in Fig. 4 (b).

On mild steel and alluminium alloys, very large striations having a wave-shaped section sometimes occur.

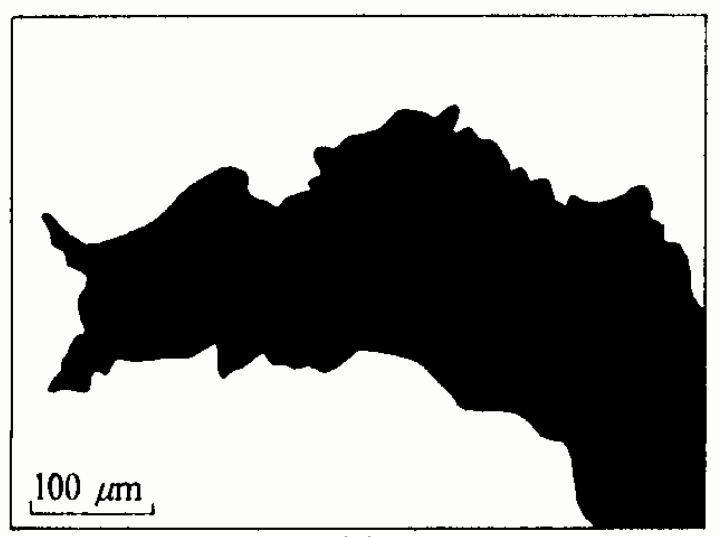

(a)

\section{The growth of fatigue cracks}

When the level of cyclic stress is sufficiently high, a microcrack will diffuse over the surface and get through the body in, by continuing to-and-fro slip processes until it has reached such the size that it is capable of propagating in the capacity of a macrocrack. That is, its growth characteristics will depend on how much it opens and closes subject to the normal cyclic stress over its faces. To get a macrocrack in a specimen at a stress level less than the plain fatigue limit of a material, some form of notch must be introduced into the specimen so that the effective length of the crack formed at the notch root is increased to a value sufficient to grow directly as a macrocrack at the applied nominal stress level. Unless expressed otherwise, it is ensues from any reference to fatigue crack growth that the crack has achieved the macrocrack period.

No accurate quantitative experimental particulars concerning the rate of fatigue crack growth had been issued prior to 1953, when Head [12] published his relationship, derivative in the abstract, between crack length and number of stress cycles. This may be owing to the fact that assessing design stresses, with respect to the fatigue properties of a material, had practically always been based on the plain fatigue limit or strength of the material being obtained from smooth laboratory specimens. Of course, the object of these design stresses was to obviate the initiation of any cracks subjected to the working loads by keeping all cyclic stresses below some critical value. The requirements to produce components or structural members of complex form that are economic, and that perform under service conditions which are not accurately defined, ended in the possibility of cracks developing even at relatively low nominal cyclic loads, for the most part as a result of fretting, or in locally highly stressed material round some discontinuity. This indicates that some components and structural members, especially those designed to have a limited life, have to function to good effect even though they may contain fatigue cracks. Forward-looking inspection procedures enabled small cracks to be detected in certain components at an early period in their expected life. However, it was given that a crack approximately $5-15 \mathrm{~mm}$ long was the smallest flaw that could be detected in the course of a routine service inspection. The need to assume the existence of cracked members in engineering construction despite the

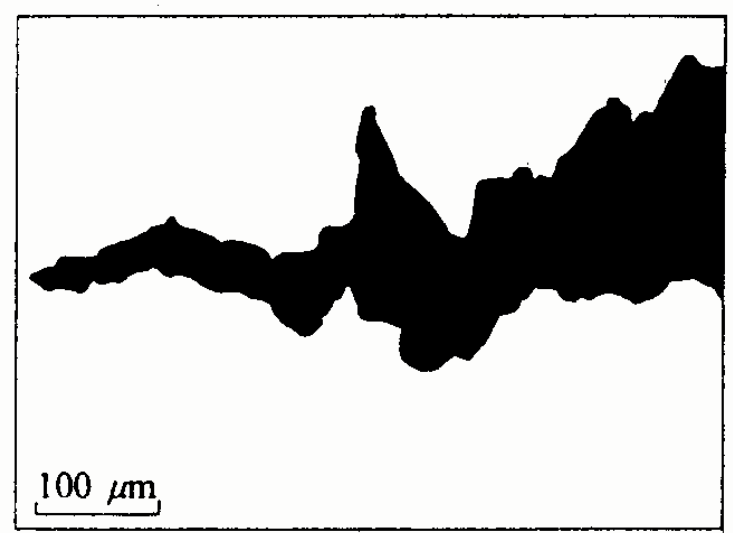

(b)

Fig. 4: Profile of crack tip in a loaded mild steel sheet 
efforts of designers to create fatigue-resistant structures is now universally accepted. These cracks will depend on the material and the values of the applied nominal mean and alternating stresses. The choice of a material from those which fulfil other necessary design factors yielding the slowest rate of crack growth for an assigned external loading will result in an increased range of safety between usual inspections. Knowledge of the growth rate behaviour of a material, together with regular inspections, may enable a cracked component to have a long service life before having to be replaced, as is shown below.

Fail-safe design (that is, a structure designed in such a way that should cracks form they will not cause catastrophic failure under the working loads until one of them reaches a known length) implies that a limiting crack length can be established which must be detected by inspection. Accordingly, designers should use all possible means to achieve the ideals of a low rate of crack propagation and high residual static strength in the presence of a crack. Probably the best way of assessing the virtues of fail-safe design is by the length of the inspection periods which it allows in relation to the importance of the design feature.

Extensive, seemingly different "laws" of fatigue crack growth have been described, and by making various acceptable assumptions some of them can be derived theoretically. All the laws can be regarded as valid in the sense that they describe a particular set of fatigue crack growth data, and they can be used to predict crack growth rates in situations similar to those used to collect the data. It is sometimes possible to fit the same set of data to apparently contradictory laws.

Since the 1940s, the problem of brittle fracture has been extensively studied.

It has been found that such low-stress (compared to the yield stress of the material) fractures always originate at flaws or cracks of various types. The fracture-mechanics approach to residual static strength in the presence of a crack makes use of the stress intensity factor $K_{\mathrm{I}}$ concept to describe the stress field at a crack tip; when $K_{\mathrm{I}}$ reaches a critical value $K_{\mathrm{C}}$ the crack extends, usually catastrophically. Values of $K_{\mathrm{I}}$ are known for a wide range of crack configurations, and the fracture-mechanics approach has proved useful in problems of material development, design, and failure analysis. In view of its success in dealing with static fracture problems, it is logical to use a similar general approach to analyze fatigue crack growth data.

In the mid $20^{\text {th }}$ century, many researchers stated how early in the fatigue life they could observe microcracks. Since then it has been clear that the fatigue life under cyclic loading consists of two phases: the crack initiation life, followed by the crack growth period until failure. This can be demonstrated in a block diagram, see Fig. 5. The crack initiation period may cover a large percentage of the fatigue life under high-cycle fatigue, i.e. under stress amplitudes just above the fatigue limit. However, for larger stress amplitudes the crack growth period can be a substantial part of the fatigue life.

An implicit special problem is how to define the transition from the initiation period to the crack growth stage.

The stress-field component $\sigma_{i j}$ at the point $(r, \theta)$ near the crack-tip is given by

$$
\sigma_{i j}(r, \theta)=\frac{K}{\sqrt{2 \pi r}} f_{i j}(\theta)+\text { other terms, }
$$

where the origin of the polar coordinates $(r, \theta)$ is at the crack tip and $f_{i j}(\theta)$ contains trigonometric functions. As the coordinate $r$ approaches zero the leading term in equation (1) dominates; the other terms are constant or tend to zero. The constant $K$ in the first term is known as the stress intensity factor.

Results for the stress concentrations of notches of very small flank angle and very small root radius $\rho$ may be used to obtain theoretical expressions for stress intensity factors. Consider a notch which, in the limit of zero root radius $(\rho)$, tends to a crack along the $y=0$ axis: if $\sigma_{\max }$ is the maximum value of $\sigma_{y y}$ at the tip, then

$$
K_{\mathrm{I}}=\frac{\sqrt{\pi}}{2} \lim _{\rho \rightarrow 0}\left(\sigma_{\max } \sqrt{\rho}\right) .
$$

Although the relationship between $K$ and $\sigma_{\max }$ is exact, the actual expression for the maximum stresses may be known only approximately. As an example of this approach consider a semi-elliptical edge notch of depth $c$ in a semi-infinite sheet subjected to a remote uniaxial tensile stress $\sigma$. Equation (2) can be written in terms of $K_{\mathrm{t}}$, the stress concentration factor (the ratio of maximum stress to applied stress), as follows:

$$
\frac{K_{\mathrm{I}}}{\sigma \sqrt{\pi c}}=\frac{K_{\mathrm{I}}}{\sigma \sqrt{\pi \ell}}=\lim _{\rho \rightarrow 0}\left\{\frac{1}{2} K_{\mathrm{t}} \sqrt{\frac{\rho}{c}}\right\},
$$

where $\ell$ is the crack length (i.e $c=\ell$ at $\rho=0$ ). The stress concentration factor $K_{\mathrm{t}}$ has been obtained for this configuration as a function of $\rho / c$. From this result for $K_{\mathrm{t}}$ and a plot of $K_{\mathrm{t}}(\rho / c)^{1 / 2} / 2$ vs $\rho / c$ (as shown in Fig. 6 ) $K_{\mathrm{I}}$ can be determined from the limit as $\rho \rightarrow 0$ and $c \rightarrow \ell$, that is,

$$
\frac{K_{\mathrm{I}}}{\sigma \sqrt{\pi \ell}}=1.13 \text {. }
$$

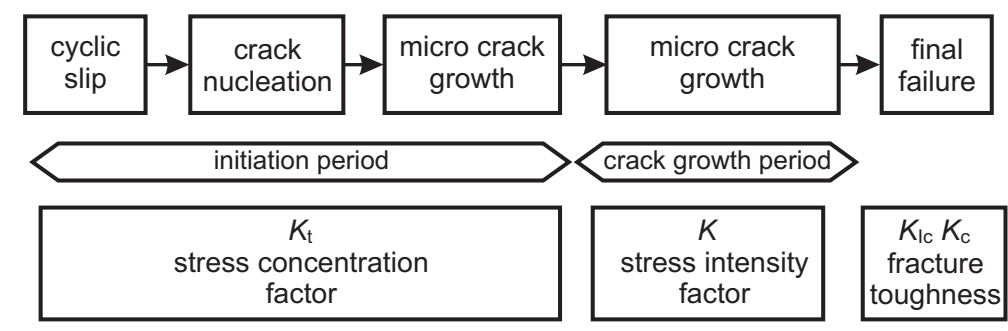

Fig. 5: Various periods of fatigue life and applicable considerations 


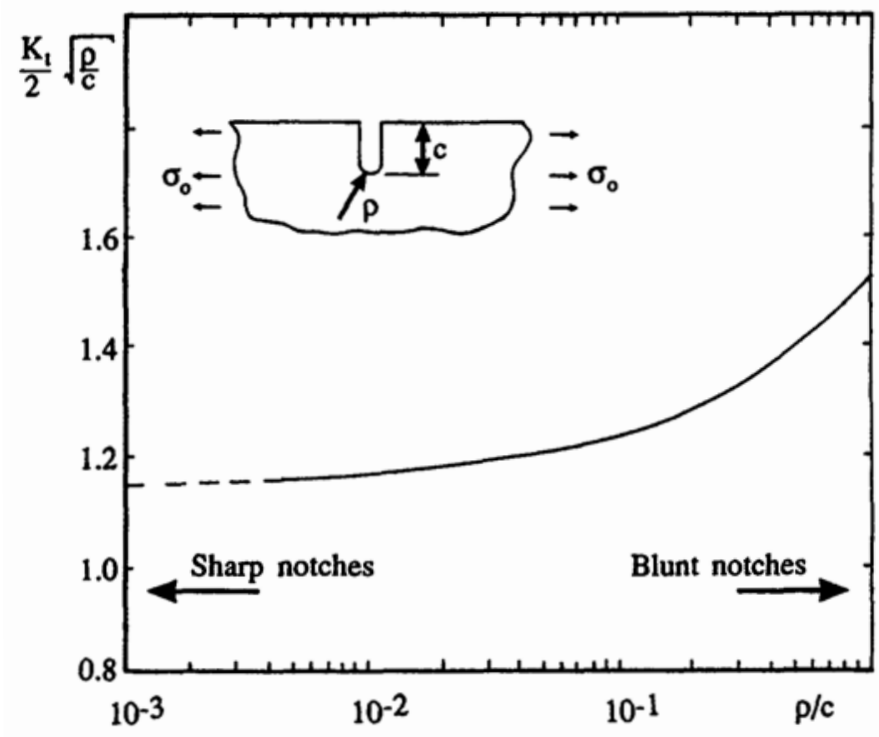

Fig. 6: Stress concentration factor as a function of notch radius

In conjunction with the well-known Paris equation, it has to be recognized that fatigue crack growth is subject to physical laws. Generally, something drives the crack extension mechanism, and this is called the crack-driving force. This force is associated with the $\Delta \mathrm{K}$-value. The stress intensity factor is related to the strain energy release rate, i.e. the strain energy in the material which is available for producing crack extension, in compliance with the expression:

$$
\frac{\mathrm{d} U}{\mathrm{~d} a}=\frac{K^{2}}{E^{*}},
$$

where $E^{*}=E$ (Young's modulus) for plane stress, and $E^{*}=E /\left(1-v^{2}\right)$ for plane strain ( $v=$ Poisson's ratio). The strain energy looks like a characteristic variable for energy balances. The experimental constants $\mathrm{C}$ and $\mathrm{m}$ in the Paris equation are not easily associated with physical properties of the material. However, the crack growth rate obtained represents the crack growth resistance of the material.

For the range of the elastic stress intensity factor, $\Delta K$, alternative parameters were developed to correlate crack propagation rates under conditions of elastoplastic crack growth, as follows: (i) crack tip plastic range, (ii) change in crack tip opening displacement, and (iii) cyclic J-integral.

As early as the 1960s it was known that the correlation of $\mathrm{d} a / \mathrm{d} N$ and $\Delta K$ depends on the stress ratio $R$. This was to be expected, because an increased mean stress for a constant $\Delta S$ should give faster crack growth while the $R$-value is also increased. Furthermore, the results of crack growth tests indicated systematic deviations of the Paris equation at relatively high and low $\Delta K$ values.

This led to the definition of three regions in $\mathrm{d} a / \mathrm{d} N-\Delta \mathrm{K}$ diagrams, namely zones I, II, and III, see Fig. 7. Evident questions are connected with the vertical asymptotes at the lower $\Delta K$ boundary of zone I and the upper $\Delta K$ boundary of zone III. The latter boundary seems to be reasoned, since if $K_{\text {max }}$ exceeds the fracture toughness (either $K_{\mathrm{c}}$ or $K_{\mathrm{Ic}}$ ), a quasi-static failure will occur and fatigue crack growth is no longer feasible. Further, it should be identified that the $K_{\max }$ value causing specimen failure in the last cycle of a fatigue crack growth test may well very from $K_{\mathrm{C}}$ or $K_{\mathrm{Ic}}$ measured in a fracture toughness experiment.

From the standpoint of fracture mechanics, the incidence of a lower boundary in region I is not so obvious. If a $K$-value can be defined for the tip of a crack, a singular stress field should be on the scene and micro-plasticity at the tip of the crack should abound. Thus, why should the crack not propagate any more; for which physical reason should there be a threshold $\Delta K$-value $\left(\Delta K_{\mathrm{th}}\right)$. New inspirations on $\Delta K_{\mathrm{th}}$ were connected with observations on so-called small cracks. These cracks occur as microcracks at the beginning of the fatigue life starting at the material surface or, more exactly, in the subsurface. The first paper on this topic was published by Pearson

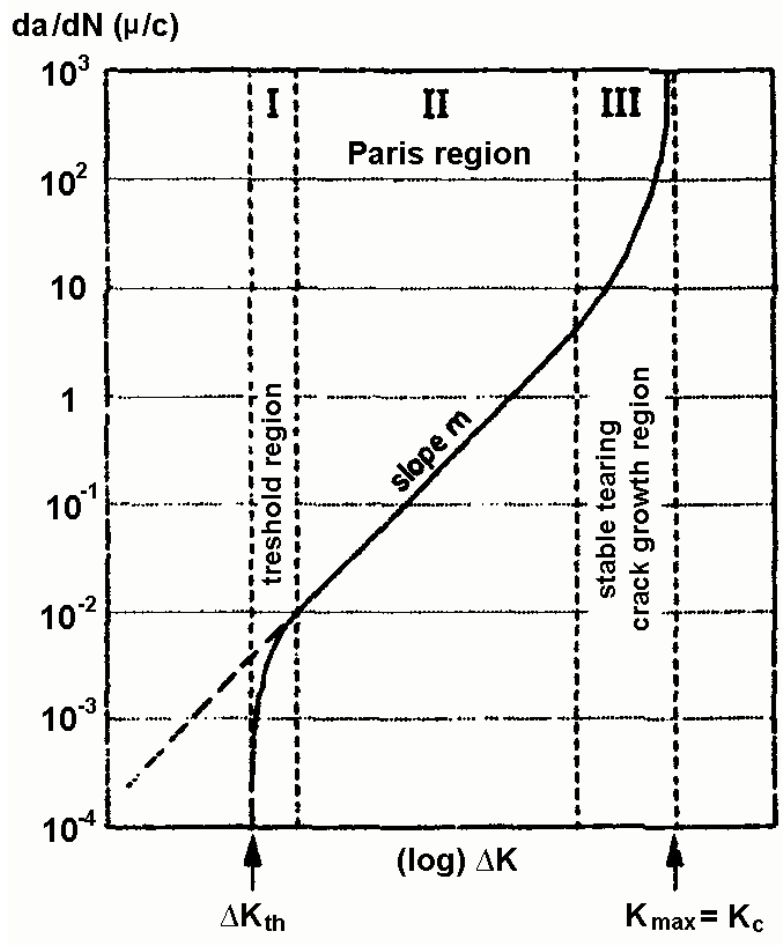

Fig. 7: Three regions of crack growth (taken from [2]) 
in 1975. He observed that small surface cracks grew more rapidly than large macro cracks at nominally similar $\Delta K$ values. Several works corroborated that microcracks could grow at low $\Delta K$-values, while macrocracks did not propagate at these low $\Delta K$-values, where $\Delta K<\Delta K_{\mathrm{th}}$. Exemplified data from Wanhill is shown in Fig. 8.

The small-crack problem became a theme for further research. Sundry crack growth obstacles extended by the material structure (e.g. grain boundaries, pearlite in steel, phase boundaries universally) could be fundamental for microcracks, whilst they were less important for macrocrack propagation. As an outcome, considerable scatter was observed in microcrack growth rates. Besides, the barriers influencing microcrack propagation could be entirely different for different materials. Although suggestions for fracture mechanics predictions of the growth of microcracks were presented, the published findings were not always convincing. Indeed, it should be noted that the $K$-conception for such small cracks in a crystalline material is made problematic. The slip band is a plastic region and its size is not small in comparison with the microcrack length.

Another issue concerning the $\Delta K_{\text {th }}$ concept involves macrocracks. Why do large cracks stop growing if $\Delta K<\Delta K_{\mathrm{th}}$ ? A formal answer to this question is because the crack driving force does not exceed the crack growth resistance of the material. At low $K$-values the crack driving force is low, which affects the crack front microgeometry. The crack front be- comes more tortuous, and the crack closure mechanism also changes. The crack driving force may just no longer be capable of producing further crack growth.

A concept to be discussed is the occurrence of crack closure, and more specifically plasticity induced crack closure. In the 1960s, Elber observed that the tip of a growing fatigue crack in an Al-alloy sheet specimen (2024-T3) could be closed at a tensile stress [13]. Crack opening proved to be a non-linear function of the applied stress, see Fig. 9. During loading from $S=0$ to $S=S_{\text {op }}$ the crack opening displacement is a non-linear function of the applied stress. The same non-linear response was observed in unloading. In the course of the non-linear behaviour the crack is partly or fully closed due to plastic deformation left in the wake of the growing crack. Elber discussed that a load cycle is only effective in driving the growth of a fatigue crack if the crack tip is fully open. Concurrently, the effective $\Delta S$ and $\Delta K$ are expressed in the form:

$\Delta S_{\text {eff }}=S_{\text {max }}-S_{\text {op }}$ and $\Delta K_{\text {eff }}=\beta \Delta S_{\text {eff }} \sqrt{\pi a}$

( $\beta$ is the geometry factor). Elber supposed that the crack growth rate is a function of $\Delta K_{\text {eff }}$ only.

$$
\frac{\mathrm{d} a}{\mathrm{~d} N}=f\left(\Delta K_{\mathrm{eff}}\right)
$$

He derived that the crack opening stress level depends on the stress ratio, for which Elber presented the relation:

$U=\frac{\Delta S_{\text {eff }}}{\Delta S}=f(R)=0.5+0.4 R$ (for 2024-T3 Al-alloy).

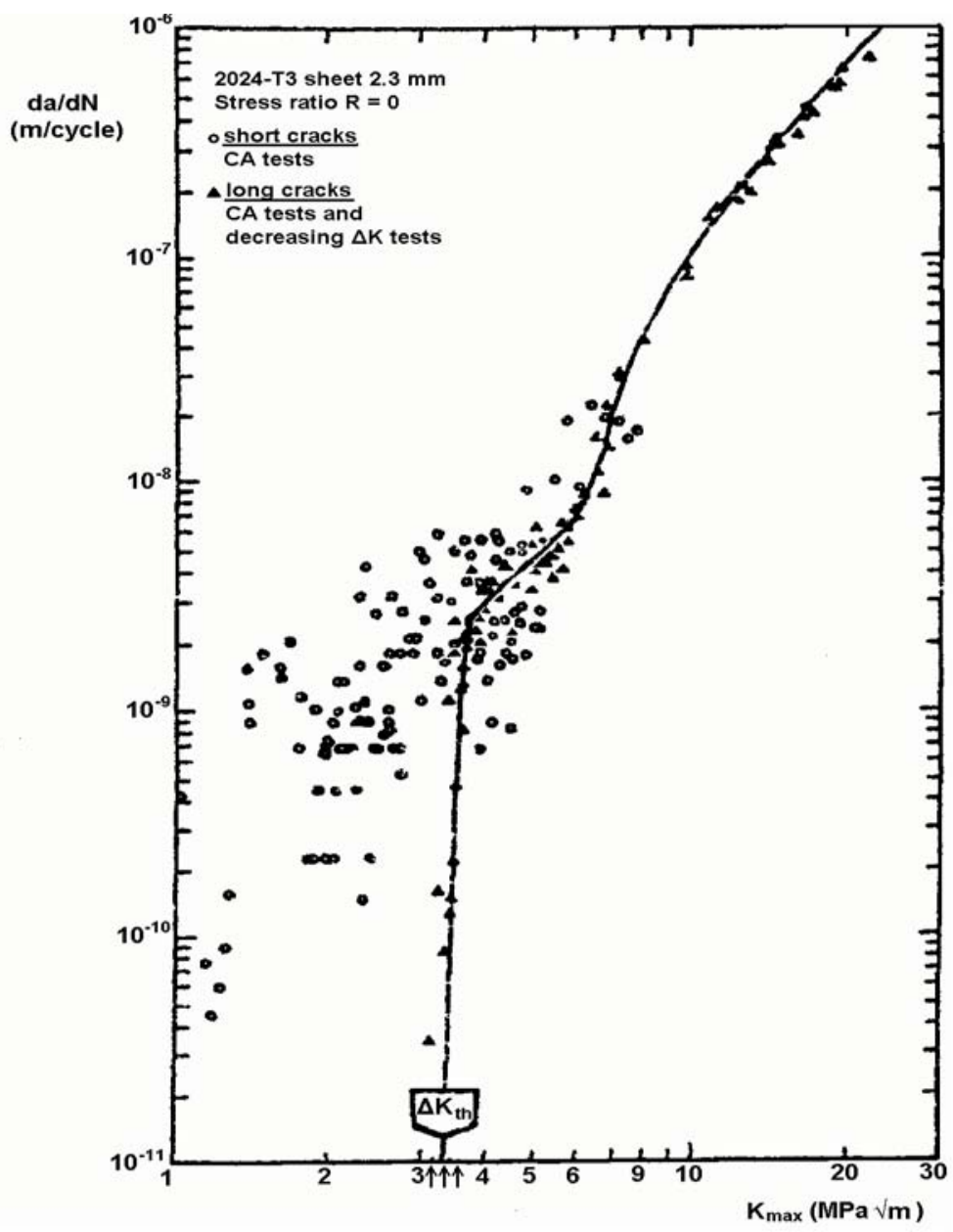

Fig. 8: Wanhill's results for large cracks and small microcracks (taken from [2]) 

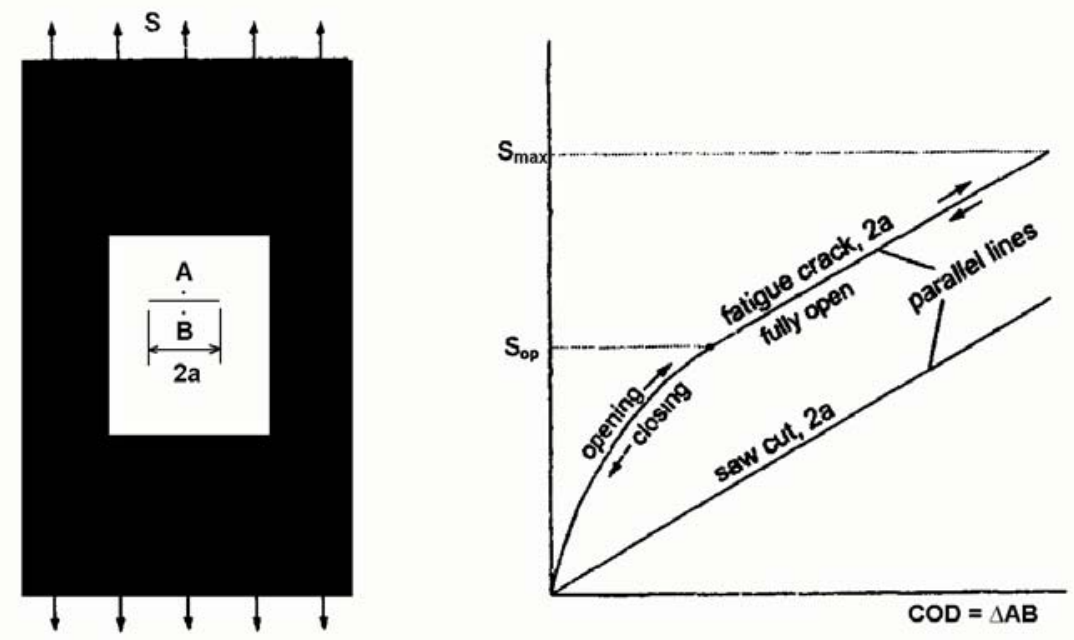

Fig. 9: Measurement of the crack opening displacement demonstrating the occurrence of plasticity induced crack closure at a positive stress (taken from [13])

In addition, he proposed that the relation should be independent of the crack length. The Elber approach continued in later investigations, partly because it was attractive to present crack growth data of a material for various $R$-values by just a single curve according to Eq. (7) It turned out that the relation in Eq. (8) could be markedly different for other materials, which is not surprising as the cyclic plastic behavior depends on the type of material.

In the 1980s, the crack closure approach was much embraced by researchers on crack growth versions for fatigue subject to variable amplitude loading.

\section{Conclusion}

The major conclusions are:

(i) fatigue failure is a product of the initiation of a crack and the follow-up propagation of this crack;

(ii) in homogeneous metals, cracks initiate at a free surface and no damage proceeds in metal far afield from this surface by cyclic stressing;

(iii) the initiation of a slip-band crack is possible only in ductile metals;

(iv) other materials can demonstrate fatigue behaviour, though this is owing to the growth of a crack from some initial flaw or fault.

\section{Acknowledgments}

This work was supported by the Grant Agency of the Czech Republic, under project No. 103/06/1382.

\section{References}

[1] Forsyth, P. J. E.: "The Application of "Fractography" to Fatigue Failure Investigations." Roy Aircraft Est, Tech Note Met, (1957), p. 257.
[2] Schijve, J.: "Fatigue of Structures and Materials in the $20^{\text {th }}$ Century. Inter Journal of Fatigue,Vol. 25 (2003), p. 679-702.

[3] Young, J. M., Greenough, A. P.: J. Inst. Metals, Vol. 89 (1960-1), p. 241.

[4] Modlen, G. F., Smith, G. C.: J. Iron Steel Inst., Vol. 194 (1960), p. 459.

[5] Ryder, D. A.: "Some Quantitative Information Obtained from the Examination of Fatigue Fracture Surfaces." Roy Aircraft Est, Tech Note Met, (1958), p. 288.

[6] Bowles, C. Q, Schijve, J.: "Crack Tip Geometry for Fatigue Cracks Grown in Air and Vacuum.” In: ASTM 811. Philadelphia , PA: American Society for Testing and Materials, 1983, p. 400-426.

[7] Forsyth, P. J. E., Ryder, D. A.: Aircraft Engineering, Vol. 32 (1960), p. 96.

[8] Pook, L. P.: Hawker Siddeley Aviation Ltd. Unpublished report, 1962.

[9] Mc Millan, J. C., Pelloux, R. M. N.: Boeing Scientific Research Lab. Document, D1-82-0558, 1966.

[10] Plumbridge, W. J., Ryder, D. A.: Acta Metall, Vol. 17 (1969), p. 1449.

[11] Frost, N. E., Holden, J., Phillips, C. E.: Crack propagation Symposium, Cranfield, 1961, p. 166.

[12] Head, A. K.: Phil. Mag., Vol. 44 (1953), p. 925.

[13] Elber W.: "Fatigue crack propagation." PhD Thesis, University New South Wales, Australia, 1968

Doc. Ing. Petr Brož, DrSc. e-mail: broz.petr@tiscali.cz

Czech Institution of Structural and Civil Engineers

Sokolská 15

12000 Prague 2, Czech Republic 